\title{
High Degree of Accuracy of a Novel Image-free Handheld Robot for Unicondylar Knee Arthroplasty in a Cadaveric Study
}

\author{
Jess H. Lonner MD, Julie R. Smith PhD, Frederic Picard MD, \\ Brian Hamlin MD, Philip J. Rowe PhD, Philip E. Riches PhD
}

Published online: 8 July 2014

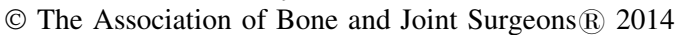

\begin{abstract}
Background Surgical robotics has been shown to improve the accuracy of bone preparation and soft tissue balance in unicondylar knee arthroplasty (UKA). However, although extensive data have emerged with regard to a CT scanbased haptically constrained robotic arm, little is known about the accuracy of a newer alternative, an imageless robotic system.
\end{abstract}

The institution of one or more of the authors (JRS, PJR, PER) received funding during the study period from Blue Belt Technologies Inc (Plymouth, MN, USA), the manufacturer of the device reported on in this study. One of the authors (FP) certifies that he, or a member of his immediate family has received or may receive payments or benefits, during the study period, an amount of less than USD 10,000. One or more of the authors of the authors (JHL, BH) certifies that he, or a member of his immediate family, has received or may receive payments or benefits, during the study period, an amount of USD 10,000 to USD 100,000 from Blue Belt Technologies Inc. One of the authors (JRS) has become an employee of Blue Belt Technologies Inc since completing the research.

All ICMJE Conflict of Interest Forms for authors and Clinical Orthopaedics and Related Research ${ }^{\mathbb{R}}$ editors and board members are on file with the publication and can be viewed on request. Clinical Orthopaedics and Related Research ${ }^{\mathbb{R}}$ neither advocates nor endorses the use of any treatment, drug, or device. Readers are encouraged to always seek additional information, including FDAapproval status, of any drug or device prior to clinical use. The authors certify that the University of Strathclyde approved the human protocol for this investigation, that all investigations were conducted in conformity with ethical principles of research, and that informed consent for participation in the study was obtained.

This work was performed at the University of Strathclyde (Glasgow, Scotland, UK) (two users) and Blue Belt Technologies Inc (Plymouth, MN, USA) (two users).

J. H. Lonner ( $\square)$

Department of Orthopaedic Surgery, Rothman Institute, Thomas Jefferson University, 925 Chestnut Street, Philadelphia, PA 19107, USA

e-mail: jesslonner@comcast.net
Questions/purposes We assessed the accuracy of a novel imageless semiautonomous freehand robotic sculpting system in performing bone resection and preparation in UKA using cadaveric specimens.

Methods In this controlled study, we compared the planned and final implant placement in 25 cadaveric specimens undergoing UKA using the new tool. A quantitative analysis was performed to determine the translational, angular, and rotational differences between the planned and achieved positions of the implants.

Results The femoral implant rotational mean error was $1.04^{\circ}$ to $1.88^{\circ}$ and mean translational error was 0.72 to $1.29 \mathrm{~mm}$ across the three planes. The tibial implant rotational mean error was $1.48^{\circ}$ to $1.98^{\circ}$ and the mean translational error was 0.79 to $1.27 \mathrm{~mm}$ across the three planes.

Conclusions The image-free robotic sculpting tool achieved accurate implementation of the surgical plan with small errors in implant placement. The next step will be to determine whether accurate implant placement translates into a clinical and functional benefit for the patient.

\section{Introduction}

Unicondylar knee arthroplasty (UKA) was introduced as a surgical treatment option for degenerative arthritis of the knee in the 1970s and now accounts for approximately $8 \%$ of knee arthroplasties [21, 26]. When only one compartment of the knee is affected, there may be a clinical and

J. R. Smith, P. J. Rowe, P. E. Riches

Biomedical Engineering Department, University of Strathclyde, Wolfson Centre, Glasgow, UK 
functional benefit to the patient in preserving bone and ligaments with UKA rather than TKA [14, 24] as well as economic benefits $[30,35]$, including reduced duration of hospitalization and rehabilitation and rapid recovery and return to work [16]. Survivorship and clinical knee scores for UKA are similar at 10 to 15 years to those reported for TKA in the hands of high-volume UKA surgeons using sound implants $[1,3,4,11,20]$. However, international registries and lower volume institutions have shown higher rates of failure at early and mid-term followup [2, 8, 21-23, $31,32]$. Higher early revision rates of up to $30 \%[8,9,15$, $19,31,33$ ] have tempered enthusiasm and limited broader use. Many failures have been shown to be related to improper patient selection, suboptimal implant or limb alignment, soft tissue imbalance, and poor designs [2, 6 , 10-12, 21-23, 27, 32].

Computer navigation has improved accuracy in UKA, but outliers still occur in as many as $40 \%$ of navigated UKAs [13]. Semiautonomous robotic technologies have further improved the accuracy of bone preparation and component alignment with a reduction in outliers compared with conventional techniques [5, 7, 17, 28, 29]. Robotic technologies were used in approximately $14 \%$ of UKAs implanted in the United States in 2012 [25]. Currently FDA-approved systems used for UKA are semiautonomous, which means that the surgeon moves the robotic instrument, but the device is preprogrammed with virtual boundaries that constrain a motorized burr from removing more bone than planned.

Initial robotic systems for UKA in the United States combine a preoperative CT scan and intraoperative mapping to register anatomic landmarks with a haptically constrained surgeon-driven robotic arm that constrains a sculpting burr within the defined space of the knee $[5,7$, 17]. A newer alternative robotic system is an image-free, surgeon-controlled handheld robotic sculpting tool that relies on intraoperative landmark mapping with safeguards achieved by controlling burr exposure and/or speed to enhance precision of bone preparation [28, 29]. Because this image-free approach is new, the accuracy of the final implant placement should be assessed for errors compared with the planned implant placement. Therefore, the purpose of this cadaveric study is to report on the accuracy of

\section{J. R. Smith}

Blue Belt Technologies Inc, Plymouth, MN, USA

\section{F. Picard}

Department of Orthopaedics, Golden Jubilee National Hospital, Clydebank, Glasgow, UK

B. Hamlin

The Bone \& Joint Center, Magee-Womens Hospital of UPMC, Pittsburgh, PA, USA the imageless semiautonomous freehand robotic sculpting system in performing bone resection and preparation in UKA.

\section{Materials and Methods}

\section{Robotic Description and Technique}

The Navio ${ }^{\mathrm{TM}}$ Precision Freehand Sculpting system (Navio ${ }^{\mathrm{TM}}$; Blue Belt Technologies Inc, Plymouth, MN, USA) is an imageless handheld robotic tool (Fig. 1). Implant planning and development of the cutting zone take place entirely intraoperatively without the need for a preoperative CT scan. The system continuously tracks the position of the patient's lower limb and the handheld robotic device using an infrared navigation system.

The system is imageless in as much as it does not use a CT or MRI to map the femoral and tibial condylar surface. It therefore relies on accurate registration of intraoperative knee kinematic assessment, anatomic landmarks, and surface mapping of the knee using a calibrated optical probe designed for use with this robotic system.

After percutaneous insertion of bicortical partially threaded pins into the proximal tibia and distal femur and attachment of optical tracking arrays, mechanical and rotational axes of the limb are determined intraoperatively by establishing the hip, knee, and ankle centers. Either the kinematic, anteroposterior (Whiteside) or transepicondylar axes of the knee are identified and selected to determine the rotational position of the femoral component. The condylar anatomy is mapped out by "painting" the surfaces with the optical probe. In this way intraoperative mapping can be completed without a preoperative CT scan. This registration process takes approximately 5 minutes on average. The intraoperative data then are used by the system's software algorithms to determine the coronal, sagittal, and axial bone axes and morphology.

A virtual model of the knee is created. Implant planning for component sizing, alignment, and volume of bone removal takes place intraoperatively (Fig. 2A). The surgeon selects the implant size that best fits the patient's anatomy and closely matches the size of the condyle to be replaced as well as its position in the coronal, sagittal, and rotational planes. Subsequent steps are directed at determining gap and ligament balance after virtual implant positioning, removal of osteophytes, and stressing of the ligaments and soft tissues. Osteophytes are excised and a dynamic soft tissue balancing algorithm is initiated. With an applied valgus stress to tension the medial collateral ligament (for medial UKA) or a varus stress to tension the lateral structures (for lateral UKA), the three-dimensional positions of the femur and the tibia are captured throughout 
a passive range of knee motion. A graphical representation of gap spacing through the range of flexion is created and determination is made regarding whether the planned position of the femoral and tibial component is adequate or

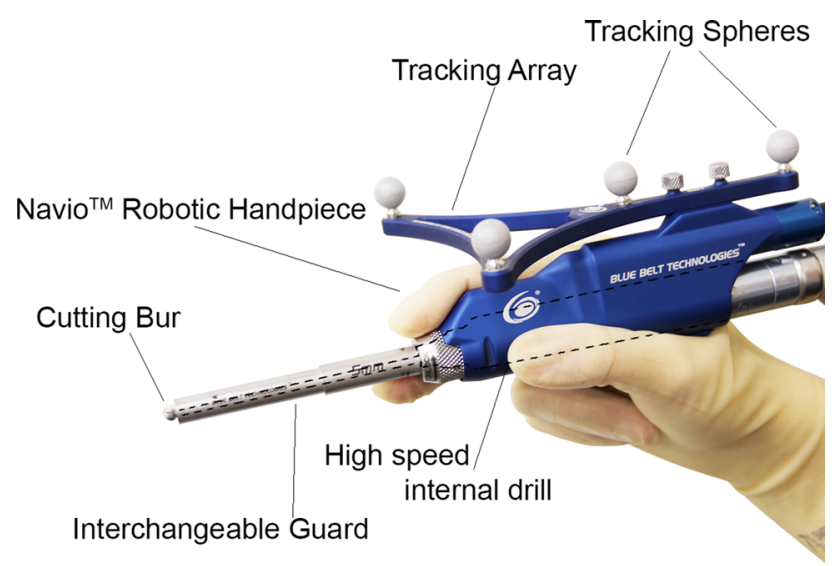

Fig. 1 The Navio ${ }^{\mathrm{TM}}$ handpiece includes a blue clamshell central unit for the user to grip, an array to allow it to be tracked by the system, and a metal guard covering the burr. Calibration of the burr to the end of the guard means that the system registers when the burr is covered by the guard or cutting. Reprinted with permission from Blue Belt Technologies Inc (Plymouth, MN, USA). adjustments can be made to achieve the desired soft tissue balance. By adjusting the implant position, including tibial slope, depth or resection, and anteriorization or distalization of the femoral component, the virtual dynamic soft tissue balance can be achieved. Adjustments in implant position and size (Fig. 2A) can be made to optimize soft tissue balance (Fig. 2B) and component tracking and position before beginning bone preparation (Fig. 2C).

Unlike predicate robotic technologies that provided haptic constraint through a robotic arm, this system works with a combination of speed and exposure control safeguards applied through a lightweight, handheld, surgeondriven semiautonomous robotic sculpting tool. In "exposure" mode, the 5- or 6-mm burr is continuously moving and is switched on and off by the user by pressing or releasing a foot pedal. A guard covers the burr, which only extends past the guard when the burr is in the "expected" cutting zone. The cutting zone is predetermined by the surgeon during the implant planning stage of the operation and the system modulates the exposure distance of the burr tip beyond the protective sheath. The position data are continuously updated in real time, resulting in fluid adjustments in the position of the burr tip. When the
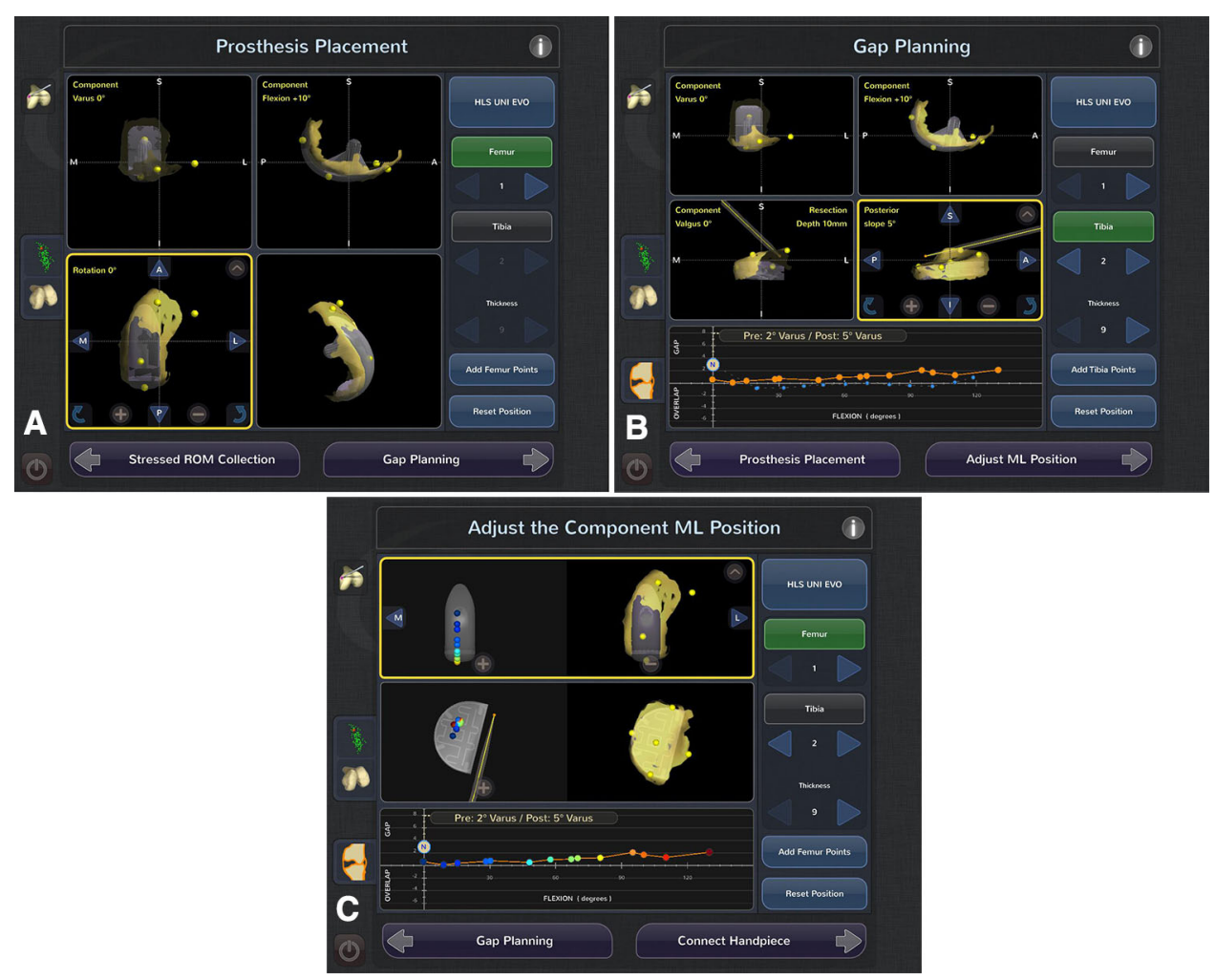

Fig. 2A-C (A) The planning stage screen shows where the user can adjust the implant size and move the position of the implant in all three planes to best match the patient's condyle. (B) The gap planning screen shows the position of the implant on the patient's condylarsurface (yellow overlay). The graph at the bottom of the screen illustrates the virtual gap balance through a range of flexion predicted from implementing the planned implant position and tensioning the ligaments. (C) Contact point screen, illustrates the contact points on both the tibial and femoral component as the knee goes through a range of flexion. 


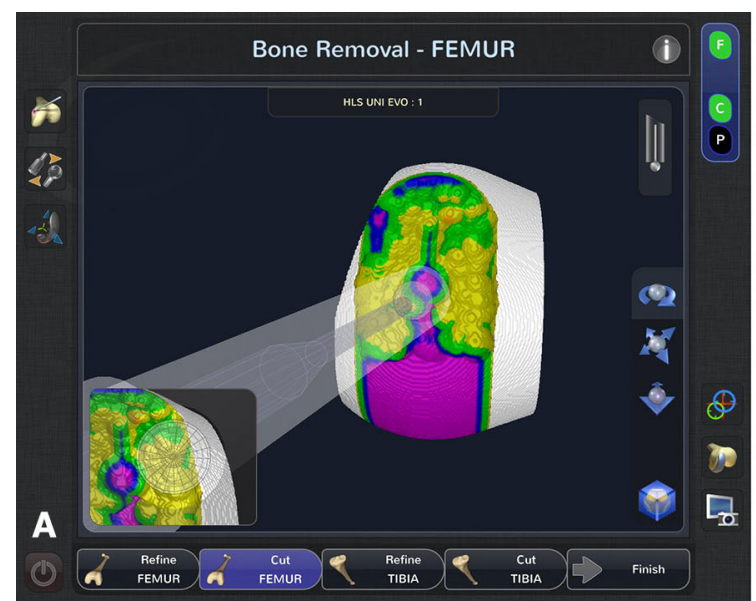

Fig. 3A-B (A) Femur and (B) tibia cutting screens show midcutting. The yellow surface is the "target" surface, green surface indicates $1 \mathrm{~mm}$ of bone still to be removed, blue surface indicates $2 \mathrm{~mm}$ of

handpiece is moved out of the cutting zone, the burr retracts within the guard. The second control mode is "speed" mode in which the burr only becomes active in the cutting zone. The speed of the rotating burr is at full power/ full speed until the intended bone is removed or it is moved beyond the desired preparation volume, at which point it linearly ramps down to zero.

After planning for size, position, alignment, bone volume, and gap balancing, the arthritic cartilage and bone are methodically removed using the handheld sculptor. The depth of bone to be removed is color-coordinated, in which the target surface is yellow, the green surface indicates $1 \mathrm{~mm}$ of bone still to be removed, the blue surface indicates $2 \mathrm{~mm}$ of bone still to be removed, and the purple layer represents $3 \mathrm{~mm}$ or more bone to be removed (Fig. 3).

\section{Validation Study}

The study was approved by the University of Strathclyde's ethics committee. In an experimental study, UKA was performed using $\mathrm{Navio}^{\mathrm{TM}}$ in 25 fresh-frozen cadavers (hemipelvis, hip to toe) donated by the Anatomy Gift Registry (Hanover, MD, USA). For consistency, all of the tests in this study used Tornier HLS UNI Evolution implants (Tornier, Montbonnot, France). All procedures were medial UKAs. The sizes of the implants were planned for the individual cadavers and therefore the implant sizes varied among cadavers.

The study was conducted by four individuals, three experienced arthroplasty surgeons (JHL, FP, BH) and one research Fellow (JRS), trained to use the system on synthetic bones (Sawbones ${ }^{\mathbb{R}}$; Pacific Research Laboratories Inc, Vashon, WA, USA) before the cadaveric validation

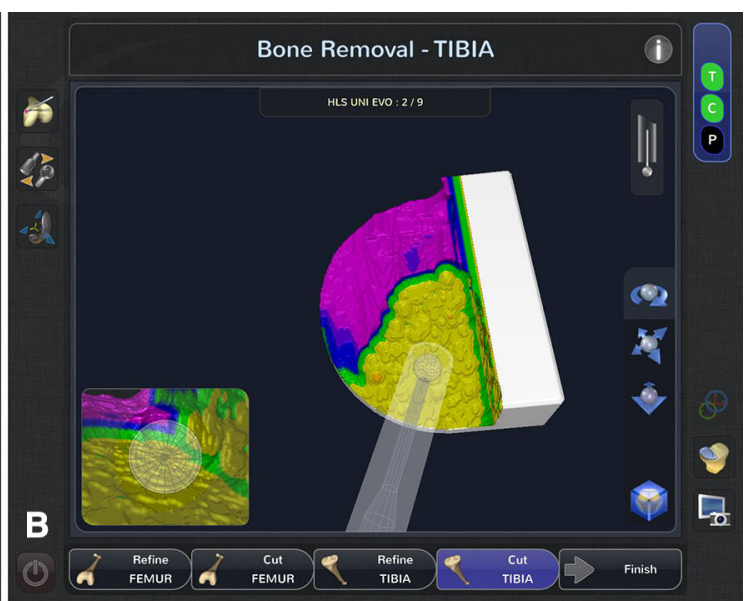

bone still to be removed, and the purple surface indicates $3 \mathrm{~mm}$ or more bone still to be removed.

study. Each user completed a different number of cadaver tests (JHL $\mathrm{n}=10$, JRS $\mathrm{n}=5$, FP $\mathrm{n}=3$, BH $\mathrm{n}=7$ ). The system was set up in a tissue laboratory in the same configuration as a typical operating room. Arrays consisting of four reflective optical markers in an asymmetric cluster were attached to partially threaded bicortical pins, which were drilled into the metaphyses of the femur and tibia. The robotic handpiece and the probes had four reflective optical markers, which were also tracked by the NDI Polaris Optical Tracking System (NDI medical, Northern Digital Inc, Ontario, Canada), which has a passive rigid body accuracy of mean 0.185 (root mean square 0.231 , SD 0.137) [34].

Bone preparation was performed per the manufacturer's recommended technique for robotic UKA with the Tornier HLS UNI Evolution implants. The femoral component, with a central lug and keel, was impacted rigidly onto the prepared bone surface and the slotted trough and peg hole on the femoral condyle optimized positioning of the component. The tibial implant in this particular design is a cemented unconstrained all-polyethylene insert. This implant design has reported good clinical and radiological results [18]. It was designed without lugs or keel to allow variable positioning on the AP axis based on intraoperative assessment of positioning relative to the femoral component. Therefore, the translational position of the tibial component on the AP axis could only be estimated. At the time this study was conducted, this was the only implant product that the system was programmed to be used with.

The main objective of the study was to assess the accuracy of the system by comparing the planned implant orientation with the actual implant orientation and report the errors calculated between the two orientations. Therefore, the positions of the implants were recorded after 
implantation using specially machined divots in the implants. A ball-point probe with optical markers was used to record the position of the divots and from this a threedimensional image of the implant position was calculated and compared with the original plan. The planned and actual cut surfaces were also compared to determine any over- or undercutting of the bone surface. The mean error and root mean square (RMS) errors were determined for each measure. The difference between the "plan" and "actual" implant position was the calculated "error." The directionality of the error was not investigated; therefore, the error values were reported as a positive value. RMS was used because the errors were positive and negative values and an average would dilute the error reported. The surgeons were not involved in the data collection or analysis.

\section{Results}

The mean recorded variances in the cut surface compared with the preoperative plan were $-0.30 \mathrm{~mm}$ (SD $0.25 \mathrm{~mm}$ ) for the femur and $-0.26 \mathrm{~mm}$ (SD $0.27 \mathrm{~mm}$ ) for the tibia (negative values represent undercutting). The RMS error was $0.67 \mathrm{~mm}$ (SD $0.37 \mathrm{~mm}$ ) and $0.61 \mathrm{~mm}$ (SD $0.29 \mathrm{~mm}$ ) for the femoral and tibial preparation, respectively.

The femoral implant angular mean error was $1.04^{\circ}$ to $1.88^{\circ}$, and the mean translational error was 0.72 to $1.29 \mathrm{~mm}$ across the three planes (Table 1). The femoral RMS error ranged from 0.88 to 2.27 . The tibial implant angular mean error was $1.48^{\circ}$ to $1.98^{\circ}$, and the mean translational error was 0.79 to $1.27 \mathrm{~mm}$ across the three planes. The tibial RMS error ranged from 0.95 to 2.43 . There were no significant differences in alignment and implant position measures or variations between surgeons or the research fellow.

\section{Discussion}

Semiautonomous robotic systems combine human expertise in surgical planning with the accuracy and reproducibility of a robotic device. They have been shown to be effective in reducing variance and improving precision in bone preparation $[5,7,17,28,29]$. Unlike its predecessors [5, 7, 17], the handheld robotic sculptor analyzed in this study does not require a preoperative CT scan. In this study, we found the accuracy of this system to be in the range of 0.8 to $1.3 \mathrm{~mm}$ of translation and $1^{\circ}$ to $2^{\circ}$ of alignment.

This study had a number of limitations. The user group consisted of three experienced orthopaedic consultants and one research fellow. Although each user

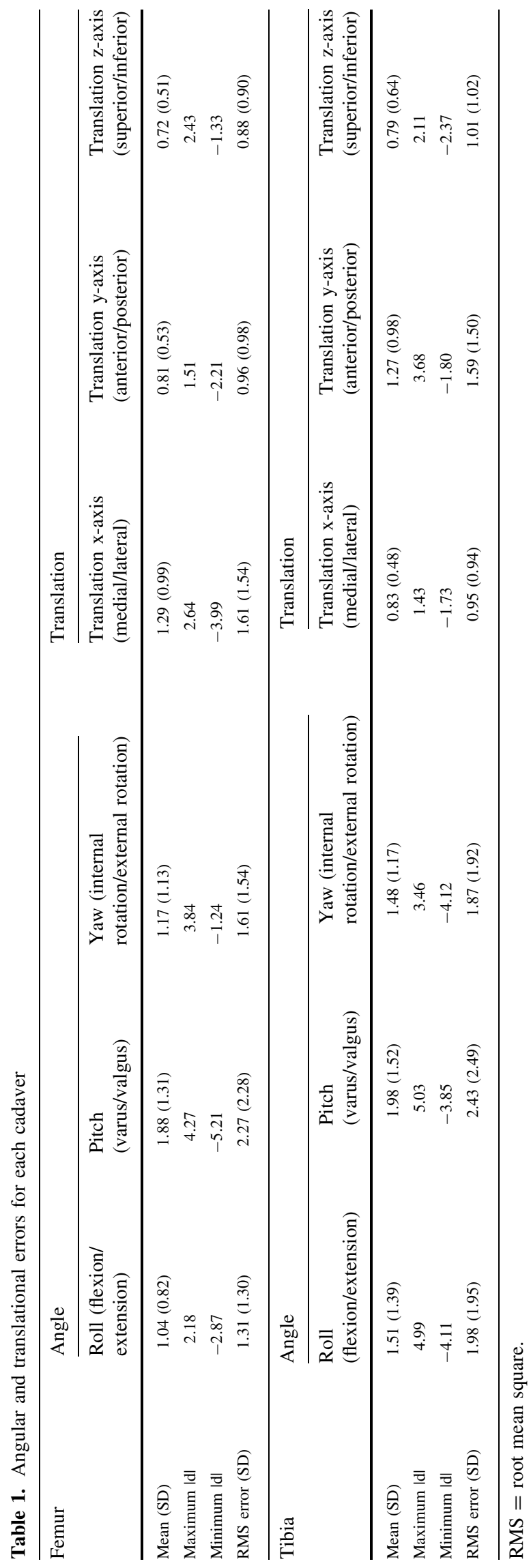


Table 2. Root mean square errors of robotic systems and conventional techniques in unicondylar knee arthroplasty

\begin{tabular}{lllll}
\hline Direction & $\begin{array}{l}\text { Navio }^{\mathrm{TM}} \text { (Blue Belt } \\
\text { Technologies Inc, Plymouth, } \\
\text { MN, USA; current study) }\end{array}$ & $\begin{array}{l}\text { MAKO Rio (MAKO } \\
\text { Surgical Corp, Fort } \\
\text { Lauderdale, FL, USA) [7] }\end{array}$ & $\begin{array}{l}\text { Acrobot } \\
\text { (MAKO Surgical } \\
\text { Corp) [5] }\end{array}$ & $\begin{array}{l}\text { Conventional } \\
\text { techniques [5] }\end{array}$ \\
\hline Flexion/extension (degrees) & 1.7 & 2.1 & 1.7 & 2.1 \\
Varus/valgus (degrees) & 2.4 & 2.1 & 3.4 & 4.1 \\
Internal/external rotation (degrees) & 1.7 & 3.0 & 1.0 & 6.3 \\
Medial/lateral (mm) & 1.3 & 1.2 & 1.8 & 2.6 \\
Anterior/posterior (mm) & 1.3 & 1.6 & 0.6 & 2.4 \\
Proximal/distal (mm) & 1.0 & 1.0 & & 1.6 \\
\hline
\end{tabular}

completed a different number of cadaver tests, there was no significant difference in the errors recorded between users. This study was completed in a laboratory using consultants and a researcher who were familiar with the system and instrumentation, although the procedures were done early in the users' learning curve. Future work is required to determine whether similar results are attainable in a clinical setting with a broad range of surgeons with varying experience with the system, robotics, and navigation for knee arthroplasty. In addition, future research will be required to determine the learning curve associated with this imageless system as well as analysis of the economic argument of whether the clinical outcome for the patient justifies the additional equipment costs.

In an initial feasibility study of this robotic system, Smith et al. [29] assessed the accuracy of bone preparation in 20 synthetic lower extremities and reported errors that were comparable with those calculated in this cadaveric study. Despite relying entirely on intraoperative surface registration and mapping, this study found that this imagefree system provides accuracy equivalent to that of earlier robotic devices (Table 2).

In the cadaveric tests performed in this study, the tibial components were screwed onto the prepared bone to rigidly secure the implant position. However, with no lugs for a corresponding post hole to indicate where the implant placement had been planned on the AP axis, translational position on the AP axis could not be considered completely accurate. Therefore, the translational error in the AP position of the tibial component could be considered a worst-case scenario. The data reported in our current study are consistent with earlier studies from other robotic systems on the market $[5,7]$ and support the hypothesis that variance of precision of bone preparation and implant placement is limited and accuracy may be improved with this robotic technology.

In conclusion, the results of this cadaveric study showed that bone preparation and implant position using this device were within a mean of $1.3 \mathrm{~mm}$ and $2^{\circ}$ of the planned implant position. Our results are comparable with those published from clinical studies investigating other semiautonomous robotic orthopedic devices [5, 7]. Future studies will determine the accuracy in clinical use compared with conventional techniques as well as functional outcomes and implant durability with this image-free robotic system, all of which are important elements of successful UKA. Certainly given the nature of this current study, these issues cannot be addressed at this time.

Acknowledgments We thank the technicians in the Anatomy Laboratory at Strathclyde University for their help during the project.

\section{References}

1. Argenson JN, Chevrol-Benkeddache Y, Aubaniac JM. Modern unicompartmental knee arthroplasty with cement: a three to ten year follow-up study. J Bone Joint Surg Am. 2002;84:2235-2239.

2. Australian Orthopaedic Association National Joint Replacement Registry. Available at: https://aoanjrr.dmac.adelaide.edu.au/en. Accessed September 13, 2013.

3. Berger RA, Meneghine RM, Jacobs JJ, Sheinkop MB, Della Valle CJ, Rosenberg AG, Galante JO. Results of unicompartmental knee arthroplasty at a minimum of ten years of follow-up. J Bone Joint Surg Am. 2005;87:999-1006.

4. Cartier P, Sanouiller JL, Grelsamer RP. Unicompartmental knee arthroplasty surgery: 10-year minimum follow-up period. $J$ Arthroplasty. 1996;11:782-788.

5. Cobb J, Henckel J, Gomes P, Harris S, Jakopec M, Rodriguez F, Barrett A, Davies B. Hands-on robotic unicompartmental knee replacement: a prospective, randomised controlled study of the Acrobot system. J Bone Joint Surg Br. 2006;88:188-197.

6. Collier MB, Eickmann TH, Sukezaki F, McAuley JP, Engh GA. Patient, implant, and alignment factors associated with revision of medial compartment unicondylar arthroplasty. J Arthroplasty. 2006;21(Suppl):108-115.

7. Dunbar NJ, Roche MW, Park BH, Branch SH, Conditt MA, Banks SA. Accuracy of dynamic tactile-guided unicompartmental knee arthroplasty. J Arthroplasty. 2012;27:803-808.e1.

8. Gioe TJ, Killeen KK, Hoeffel DP, Bert JM, Comfort T, Scheltema K, Mehle S, Grimm K. Analysis of unicompartmental knee arthroplasty in a community-based implant registry. Clin Orthop Relat Res. 2003;416:111-119.

9. Gioe TJ, Novak C, Sinner P, Ma W, Mehle S. Knee arthroplasty in the young patient: survival in a community registry. Clin Orthop Relat Res. 2007;464:83-87. 
10. Hamilton WG, Collier MB, Tarabee E, McAuley JP, Engh CA Jr, Engh GA. Incidence and reasons for reoperation after minimally invasive unicompartmental knee arthroplasty. J Arthroplasty. 2006;21(Suppl):98-107.

11. Heck DA, Marmor L, Gibson A, Rougraff BT. Unicompartmental knee arthroplasty: a multicenter investigation with long-term follow-up evaluation. Clin Orthop Relat Res. 1993;286:154-159.

12. Hernigou P. Deschamps G. Alignment influences wear in the knee after medial unicompartmental arthroplasty. Clin Orthop Relat Res. 2004;423:161-165.

13. Jenny JY, Boeri C. Unicompartmental knee prosthesis implantation with a non-image-based navigation system: rationale, technique, case-control comparative study with a conventional instrumented implantation. Knee Surg Sports Traumatol Arthrosoc. $2003 ; 11: 40-45$.

14. Laurencin CT, Zelicof SB, Scott RD, Ewald FC. Unicompartmental versus total knee arthroplasty in the same patient. Clin Orthop Relat Res. 1991;273:151-156.

15. Lewold S, Robertsson O, Knutson K, Lidgren L. Revision of unicompartmental knee arthroplasty Outcome in 1,135 cases from the Swedish Knee Arthroplasty study. Acta Orthop Scand. 1998;69:469-474.

16. Lombardi AV, Berend KR, Walter CA, Aziz-Jacobo J, Cheney NA. Is recovery faster for mobile bearing unicompartmental than total knee arthroplasty? Clin Orthop Relat Res. 2009;467: $1450-1457$.

17. Lonner JH, John TK, Conditt MA. Robotic arm-assisted UKA improves tibial component alignment: a pilot study. Clin Orthop Relat Res. 2010;468:141-146.

18. Lustig S, Paillot JL, Servien E, Henry J, Ait Si Selmi T, Neyret P. Cemented all polyethylene tibial insert unicompartmental knee arthroplasty: a long term follow-up study. Orthop Traumatol Surg Res. 2009;95:12-21.

19. Marmor L. Unicompartmental arthroplasty of the knee with a minimum of 10 year follow up. Clin Orthop Relat Res. 1988; 228:171-177.

20. Murray DW, Goodfellow JW, O'Connor JJ. The Oxford medial unicompartmental arthroplasty: a ten-year survival study. $J$ Bone Joint Surg Br. 1998;80:983-989.

21. National Joint Registry. Available at: www.njrcentre.org.uk/ njrcentre/Portals/0/Documents/England/Reports/10th_annual_ report/NJR\%2010th\%20Annual\%20Report\%202013\%20B.pdf. Accessed April 24, 2014.
22. New Zealand Joint Registry. Available at: www.nzoa.org.nz/ news/new-zealand-joint-registry-thirteen-year-report. Accessed September 13, 2013.

23. Norwegian Arthroplasty Register. Available at: http://nrlweb. ihelse.net/eng/Report_2010.pdf. Accessed September 13, 2013.

24. Noticewala MS, Geller JA, Lee JH, Macaulay W. Unicompartmental knee arthroplasty relieves pain and improves function more than total knee arthroplasty. J Arthroplasty. 2012;27(Suppl): 99-105.

25. Orthopedic Network News. 2013 Hip and Knee Implant Review. Available at: www.OrthopedicNetworkNews.com. 2013;24. Accessed September 14, 2013.

26. Riddle DL, Jiranek WA, McGlynn FJ. Yearly incidence of unicompartmental knee arthroplasty in the United States. J Arthroplasty. 2008;23:408-412.

27. Ridgeway SR, McAuley PJ, Ammeen DJ, Engh GA. The effect of alignment of the knee on the outcome of unicompartmental knee replacement. J Bone Joint Surg Br. 2002;84:351-355.

28. Smith JR, Picard F, Rowe PJ, Deakin A, Riches PE. The accuracy of a robotically-controlled freehand sculpting tool for unicondylar knee arthroplasty. J Bone Joint Surg Br. 2013;95(Suppl):68.

29. Smith JR, Riches PE, Rowe PJ. Accuracy of a freehand sculpting tool for unicondylar knee replacement. Int J Med Robot. 2013 Aug 11 [Epub ahead of print].

30. SooHoo NF, Sharifi H, Kominski G, Lieberman JR. Cost effectiveness analysis of unicompartmental knee arthroplasty as an alternative to total knee arthroplasty for unicompartmental osteoarthritis. J Bone Joint Surg Am. 2006;88:1975-1982.

31. Squire MW, Callaghan JJ, Goetz DD, Sullivan PM, Johnston RC. Unicompartmental knee replacement: a minimum 15 year follow up study. Clin Orthop Relat Res. 1999;367:61-72.

32. Swedish Knee Arthroplasty Register. Available at: www.knee. nko.se/english/online/uploadedFiles/117_SKAR_2012_Engl_1.0. pdf. Accessed September 13, 2013.

33. Thornhill TS, Scott RD. Unicompartmental total knee arthroplasty. Orthop Clin North Am. 1989;20:245-256.

34. Wiles AD, Thompson DG, Frantz DD. Accuracy assessment and interpretation for optical tracking systems. Medical Imaging. 2004;5367:421-432.

35. Willis-Owen CA, Brust K, Alsopa H, Miraldo M, Cobb JP. Unicondylar knee arthroplasty in the UK National Health Service: an analysis of candidacy, outcome and cost efficacy. Knee. $2009 ; 16: 473-478$. 